\title{
MODELLING AND SIMULATION OF HUMAN-CENTRED ASSEMBLY SYSTEMS - A REAL CASE STUDY
}

\author{
Anna M. Lassila, Sameh M. Saad ${ }^{*}$ and Terrence Perera \\ School of Engineering, Sheffield Hallam University, UK \\ "email:s.saad@shu.ac.uk
}

Tomasz Koch and Jaroslaw Chrobot Institute of Production Engineering and Automation, Wroclaw University of Technology, POLAND

\begin{abstract}
This paper reports the experiences gained through the use of computer simulation in the modelling of a human-centred assembly line in an automotive manufacturing company. The main themes of the paper include the problem of achieving sufficient accuracy in the description of human operations, the difficulties encountered in data collection, and the modelling of human-centred operations. The importance of an accurate representation of human behaviour for the validity of the developed simulation model in a human-centred system is discussed. Finally, the simulation results and some recommendations for system improvement are presented.
\end{abstract}

\section{INTRODUCTION}

In today's highly competitive environment, companies are under constant pressure to improve their production processes. However, correct actions and decisions require accurate information about the system performance. Since the behaviour of many complex systems changes over a period of time, a technique such as computer simulation is necessary for building valid models and generating accurate performance data.

Simulation is a widely used tool for building models of real or proposed systems in order to evaluate their performance in dynamic conditions. The output data of the simulation can be used to identify system bottlenecks and to generate alternative states that may provide the desired performance improvements for the system. The major advantage of simulation is that it enables experiments with the system without disturbing the operations of the real process (Kelton et al., 2004).

Traditionally, system modelling and simulation has concentrated on the technological aspects of the systems (Ehrhardt, 1994) whereas the workers have simply been modelled as resources that perform simple and clearly defined tasks with time dependent availability and varying efficiency (Baines and Kay, 2002). While this approach is sufficient for highly mechanised processes (Ehrhardt, 1994), it can seriously distort the capacity predictions of systems with high human work 
content (Baines, 2003). Since the competitiveness of many manufacturing companies still depend on the flexibility and responsiveness of humans (Baines and Kay, 2002), an increasing number of researchers have in recent years attempted to develop methods for a more accurate modelling of human behaviour, e.g. Ehrhardt et al. (1994), Cacciabue (1998), Schmidt (2000), Baines and Kay (2002), Baines et al. (2003) and Brailsford and Schmidt (2003). Throught (2004) argued against this trend stating that the non-deterministic behaviour of people is a result of individual characters, opinions, needs and requirements that cannot be modelled probabilistically without a high level of simplification.

In this paper, the problem of accurate modelling of assembly systems with high human work content is highlighted in a case study of a human-centred assembly line in an automotive manufacturing company. The problems encountered during the development of the simulation model using discrete event simulation software Arena are reported. These range from data collection to model validation. According to Jayaraman and Gunal (1997) discrete event simulation is now a standard tool in the automotive industry.

\section{LITERATURE REVIEW}

Over the last two decades, fully automated factories that would not have to consider the cost or variation problems caused by human involvement have been the ultimate goal of many companies (Braun et al, 1996). However, demand for skilled workers has increased simultaneously with growing automation levels (Arai et al., 1997). This was caused by the increasing complexity and customisation of products, shorter product life cycles and low and unpredictable demand, creating production conditions that can best be dealt with by flexible workers. Even flexible, technologically advanced manufacturing systems relay heavily on the skills of workers (Hitomi, 1996).

Consequently, in recent years academic interest in human factors and their influence on manufacturing system performance has intensified. Sociological insights, for instance on the role of the conditions in which people work (e.g. Bonnes and Secciaroli, 1995) and the characteristics and behaviour of individuals (e.g. Furnham, 1992), are often found to be relevant to manufacturing system development. Hence, current manufacturing research activity is concerned with how to properly include these factors into the system development process e.g. system modelling and simulation.

One of the first detailed methodologies for including human factors in simulation models was proposed by Ehrhardt et al. (1994). Ehrhardt considered strain and stress factors of different manual tasks and their influence on human performance. The significance of human behaviour on manufacturing system performance was emphasised in a study by Fan and Gassmann (1995). They noted that the performance of a manufacturing cell depended greatly on the person working on the cell regardless of the automation level of the cell. Cacciabue (1996) emphasised the importance of a good working environment analysis and of the collection of appropriate information for successful modelling of man-machine interactions.

Later, Schmidt (2000) argued that even though human behaviour is very complex, it can, within limits, be modelled and thus can become deterministic. He 
proposed a general reference model for modelling human behaviour in any application. His model was applied by Brailsford and Schmidt (2003) to a health care system. Baines and Kay (2002) proposed a methodology for modelling highly simplified relationships between workers, their environment and their subsequent performance. Baines et al. (2003), on the other hand, connected an external human performance model with a discrete event simulation tool in order to study the influence it can have to the performance of a system.

\section{CASE STUDY FROM AUTOMOTIVE INDUSTRY}

The complexity of trying to model human work with sufficient accuracy was encountered during a simulation and development project of a human-centred assembly line in an automotive manufacturing company. The experiments carried out during the project are shortly described in this section.

\subsection{System description}

The automotive manufacturing plant, located in Eastern-Europe, has two very similar manual un-phased assembly lines for producing two of their products (A and B), which are produced in more than 300 different versions. The assembly lines, presented in Figure 1, operate independently and differ from each other only by stations 13 and 14, which are present on line 2 only. Consequently, line 1 is unable to process one version of product $\mathrm{A}$. This study mainly examines line 2 .

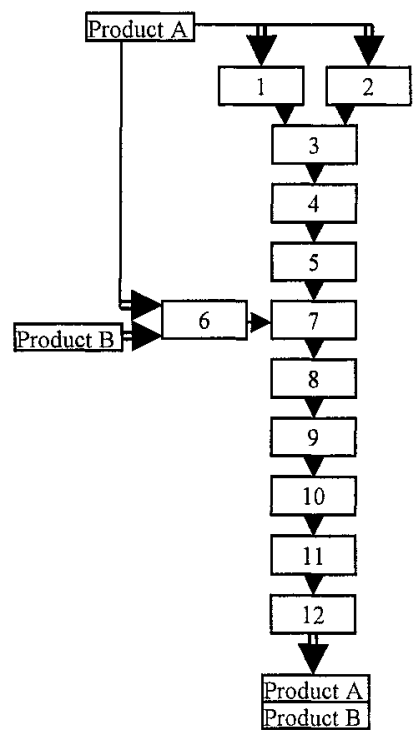

LINE 1

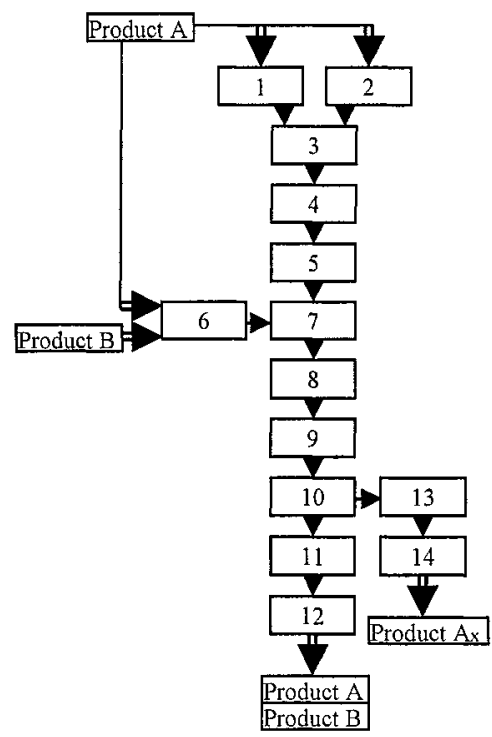

LINE 2

Figure 1 - Assembly lines

Normally, the assembly of products A require work on all stations on the line, 
except on stations 13 and 14 . Those resources are only used by a special version of product A. P roducts B, on the o ther hand, use only half of the line s tarting from station 6 and finishing at station 12. The products are processed in batches according to the orders. Any batch size is possible, starting from only one product up to hundreds. Machines need to be reset every time the product version changes. The length of the changeover depends on the similarity of two consecutive products.

Each station has one operator who performs the assigned tasks either fully manually or with the use of simple machines at some stations. A major part of the work on the line is performed manually as can be seen in Figure 2, which illustrates the estimated manual work content at each station.

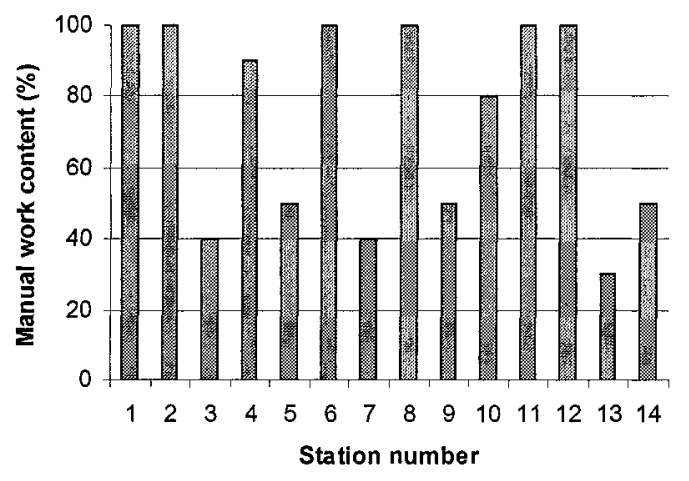

Figure 2 - Estimated manual work content of the stations on assembly line 2

Currently, the workload is not fully balanced between the stations and the processing of a part on consecutive stations may require different times. For this reason, small buffers have been built between stations to hold 5 to 10 products. The flow is improved by encouraging operators to move between the stations to prevent starving or blocking on the line.

\subsection{Data collection and process observations}

In the past the company had collected very little data about the line performance, and no real measurements of the system had been conducted. However it was estimated that the current line output rate is one product in every $80+$ seconds, while the line had been designed for a 44 seconds cycle time. Typically, the system produces around $330 \mathrm{~A}$ products or $350 \mathrm{~B}$ products in one eight hour shift on one line. The estimated average uptime of a line is $85 \%$ and the amount of products requiring rework is around $2 \%$. The setup time between products can reach anything up to 15 minutes.

Since the existing data was not sufficient for the simulation model development, the requirements for additional data were recognised and the measurements were performed on the line. Since the number of product variations assembled on the system exceeded 300 and many of them had very similar processing requirements, ten different products were selected to represent all products processed on the line. The measurements captured the processing, transfer, setup and rework times for each product at each station. 
During these measurements the general functioning of the line was observed. In overall, the line appeared very unorganised. Big containers of materials were waiting to be used, most of the buffers were full with unfinished products, people were continuously moving between the stations, regularly a group of people gathered around a machine that was not functioning correctly, and while some operators were working very hard others were idle while waiting for work to be completed somewhere else on the line. In addition, there was an apparent lack of balance in the workload of the stations and standardised work practices. Since most of the work on the line was performed manually by the operators, a large number of people were constantly present and moving around in a relatively small area as encouraged by the management.

The collected data revealed a high variation at least on one station in all time measurements. No particular station was consistently recognised as having a higher variation than others for all products. Figure 3 illustrates the processing time variation for one of the measured products. It indicates that the variation a t most stations are just within acceptable limits, however the mode values still vary strongly among the stations. We suspect that this variation was to some degree caused by differently skilled and motivated operators.

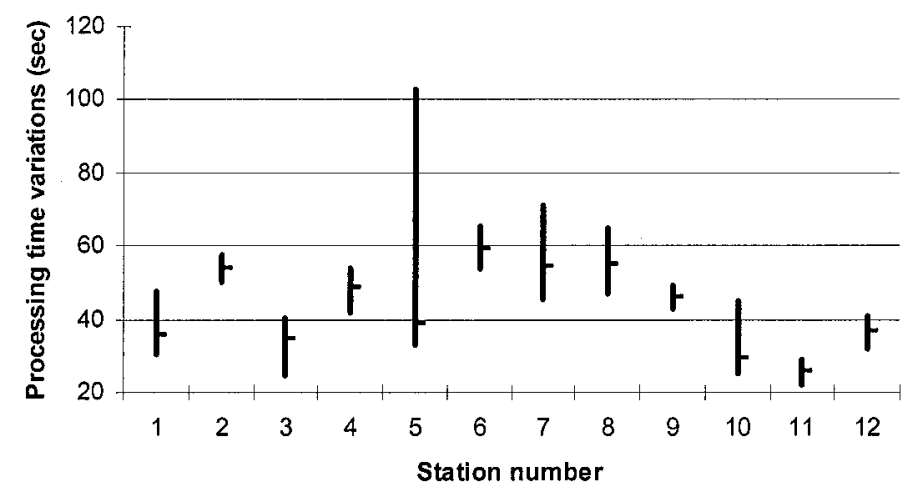

Figure 3 - Processing time variations ( $m i n, \max$, and mode) on assembly line 2 for one product type

\subsection{Modelling human-centred processes}

The main question e ncountered during model development was how to model these human-centred processes. The decision was made to represent each operator as a process with limited capacity and scheduled availability. The processing times were included as triangular distributions with the mode calculated over the sample space of the normal values, where normal values were defined as values that regularly occurred during the measurements.

In addition, a separate random breakdown operator was created to model the temporary unavailability of operators caused by off-station work tasks or not work related activities. These activities had a significant influence on the performance of the system as they regularly disturbed the normal functioning of the line by causing starvation and blockage between stations. The effects of running the simulation 
model with and without the random breakdown operator are illustrated in the next section.

\subsection{Model validity and simulation results}

The validity of the developed simulation model was evaluated by comparing the performance of the model to the real system. Three separate tests were carried out, the results of which are presented in Table 1. The output and cycle time values obtained from the simulation model were found to be very similar to the estimated values of the real system, differing at most $10.9 \%$. Therefore the tests are suitable for system analysis and experimentation.

Table 1 - Simulation model validation test results

\begin{tabular}{|c|c|c|c|c|c|c|c|c|}
\hline \multirow{2}{*}{$\begin{array}{c}\text { Test } \\
\text { no. }\end{array}$} & Description & \multirow{2}{*}{$\begin{array}{c}\text { No. of } \\
\text { setups }\end{array}$} & Output & $\begin{array}{c}\text { Cycle } \\
\text { time } \\
\text { (sec) }\end{array}$ & Output & $\begin{array}{c}\text { Aycle } \\
\text { time } \\
\text { (sec) }\end{array}$ & $\begin{array}{c}\text { in } \\
\text { output }\end{array}$ & $\begin{array}{c}\text { in } \\
\text { cycle } \\
\text { time }\end{array}$ \\
\hline $\mathbf{1}$ & Batch of A's & 1 & $\sim 330$ & $\sim 80$ & 345 & 80 & 4.5 & 0 \\
\hline $\mathbf{2}$ & Batch of B's & 1 & $\sim 350$ & $\sim 80$ & 372 & 74 & 6.3 & 8.1 \\
\hline $\mathbf{3}$ & $\begin{array}{c}\text { Production } \\
\text { plan (a mix of } \\
\text { A's and B's) }\end{array}$ & 3 & $\sim 310$ & $\sim 80$ & 311 & 88.7 & 0.3 & 10.9 \\
\hline
\end{tabular}

A lead time analysis using the developed simulation model was performed for the same test cases previously used in the validation test. In addition, a fourth test was run using the production plan of test case 3 but removing the random breakdown operators. The results are presented in Table 2.

Table 2 - Lead time analysis using the developed simulation model

\begin{tabular}{|c|c|c|c|c|}
\hline $\begin{array}{c}\text { Test } \\
\text { no. }\end{array}$ & $\begin{array}{c}\text { Average lead } \\
\text { time (min) }\end{array}$ & $\begin{array}{c}\text { Average } \\
\text { processing } \\
\text { time (min) }\end{array}$ & $\begin{array}{c}\text { Maximum } \\
\text { transfer time } \\
\text { (min) }\end{array}$ & $\begin{array}{c}\text { WAITING } \\
\text { (min) }\end{array}$ \\
\hline $\mathbf{1}$ & 39 & 8.6 & 1 & $\mathbf{2 9 . 4}$ \\
\hline $\mathbf{2}$ & 26.7 & 4.5 & 1 & $\mathbf{2 1 . 2}$ \\
\hline $\mathbf{3}$ & 33.4 & 6.6 & 1 & $\mathbf{2 5 . 8}$ \\
\hline 4 & 28.5 & 6.6 & 1 & $\mathbf{2 0 . 9}$ \\
\hline
\end{tabular}

The results indicate that the temporary unavailability of operators and the machine downtimes together increase the average lead time by around 5 minutes. However, even without the breakdowns waiting in the queues still constitute a major part of the lead-time as can be seen from Table 2. While the average actual processing time for a product during a mixed production is only 6.6 minutes, the average lead-time is over 28 minutes. Therefore, on average, a product spends more than twenty minutes waiting in the buffers between the stations, accounting for more than $70 \%$ of the total lead-time. The set-ups that occurred during the last two tests increased the lead-times by only a few seconds.

The long waiting times and persistent high utilisation of the buffers were revealed during the system simulation. Since all buffers had limited capacities, they regularly blocked the upstream processes causing chaos on the line. Figure 4 shows that for products $A$ the buffers at the beginning of the line were full for almost all the time, while the B products filled the output buffers of stations 6 and 7 . The 
average utilisation of resources was around $62 \%$. The utilisation of the resources was clearly higher at the middle of the line. Blocking prevented utilisation to be increased.

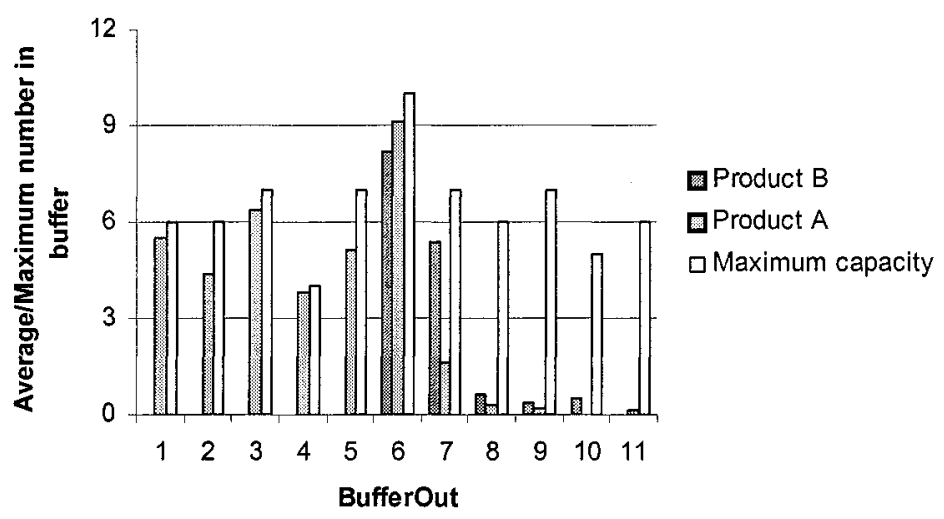

Figure 4 - Average number of products in the buffers compared to the maximum capacities of the buffers

The results suggest that the high variation in the processing times caused by a general lack of organisation and standardisation concerning manual activities resulted in a highly unstable system. The high content of manual work on the assembly line increased the system variation, because the work of the operators was not time-sequenced by the machine times and did not follow defined procedures.

The buffers around these human-centred stations increased the processing time variation even further, since they stopped the continuous flow of the parts and broke the direct connection between consecutive stations. As a consequence, the timesequencing force of the preceding and following stations was lost. The stations became isolated islands without a common rhythm, and each station was working for its own buffer rather than for the next station. Gradually, the whole line slowed down and the product lead times increased.

To improve the system, first the actual work content of each station needs to be clarified, standardised and measured so that the work load can be rebalanced among the stations in order to minimise variation. Next, the work practices including the operator movements between stations and the system layout should be critically reviewed. In the current chaotic line conditions, the recommendation from system simulation to add a machine to station 7 would not address the actual problem.

\section{CONCLUSIONS}

This paper has reported on the experiences gained during the simulation model development in an automotive manufacturing company. The paper focused on the human modelling issues from data collection to defining sufficient accuracy to operators. Data collection and simulation revealed a chaotic system with high time variation in every processing stage. The immediate actions for line performance 
improvement should address organisational practices rather than capacity increases. In order to reduce system idle time and improve utilisation a full line balancing study needs to be carried out. However, at this stage, two types of scheduling scenarios, backward scheduling before the bottleneck station 7 and forward scheduling beyond this station, has been recommended.

The chaotic conditions on the assembly line were caused by the lack of proper planning and control. Appropriate control measures are especially important in systems with high human work content to manage the additional variation human operators create. The rather simplistic modelling approach of humans used in this study provided accurate data as the system itself was chaotic. However, if the product flows were controlled better, the individual characteristics of the humans would become more influential on system performance and a more detailed representation of operator characteristics may be required.

\section{REFERENCES}

1. Arai E, Shirase K, Wakamatsu H, Murakami Y, Takata M, Uchiyama N. "Role of production simulation for human oriented production systems" In the Proceedings of $14^{\text {th }}$ International conference on Production Research. 1997: 758-761.

2. Baines TS, Kay JM. "Human performance modelling as an aid in the process of manufacturing system design: a pilot study" In the International Journal of Production Research. 2002; 40 (10): 2321 2334.

3. Baines T, Mason S, Siebers PO, Ladbrook J. "Humans: the missing link in manufacturing simulation?" In the Simulation Modelling Practice and Theory, 2003.

4. Bonnes M, Secciaroli G. Environmental psychology: a psycho-social introduction. Sage Publications, 1995.

5. Brailsford S, Schmidt B. "Towards incorporating human behaviour in models of health care systems: An approach using discrete event simulation" In the European Journal of Operational Research. 2003; 150: 19-31.

6. Braun WJ, Rebollar R, Schiller EF. "Computer aided planning and design of manual assembly systems" In the International Journal of Production Research. 1996; 34 (8): 2317-2333.

7. Cacciabue PC. "Understanding and modelling man-machine interactions" In the Nuclear Engineering and Design. 1996; 165: 351-358.

8. Cacciabue PC. Modelling and Simulation of Human Behaviour in System Control. Springer-Verlag Berling and Heidelberg GmbH\&Co, 1998.

9. Ehrhardt I, Herper H, Gebhardt H. "Modelling strain of manual work in manufacturing systems" In the Proceedings of the 1994 Winter Simulation Conference. Orlando, Florida. 1994: 1044-1049.

10. Fan IS, Gassmann R. "Study of the practicalities of human centred implementation in a British manufacturing company" In the Computer Integrated Manufacturing Systems. 1995; 8 (2): 151154.

11. Furnham A. Personality at work: the role of individual differences in the workplace. Routledge, 1992.

12. Hitomi K. "Manufacturing excellence for 21 st century production" In the Technovatio. 1996; 16 (1): $33-41$.

13. Jayaraman A, Gunal AK. "Application of discrete event simulation in the design of automotive powertrain manufacturing systems" In the Proceedings of the 1997 Winter Simulation Conference. Atlanta, Georgia, USA. 1997: 758-764.

14. Kelton WD, Sadowski RP, Sturrock DT. Simulation with Arena. 3rd Ed. New York: McGraw-Hill Companies, 2004.

15. Schmidt B. "The Modelling of Human Behaviour" Online in http://www.or.unipassau.de/english/2/Human_Factors.pdf, 2000.

16. Trought B. "Why pcople are a manufacturing problem" To appear in the Proceedings of the 2 nd International conference on Manufacturing Research. Sheffield, UK. September 2004. 